\title{
Esophageal perforation after epicardial ablation: an endoscopic approach
}
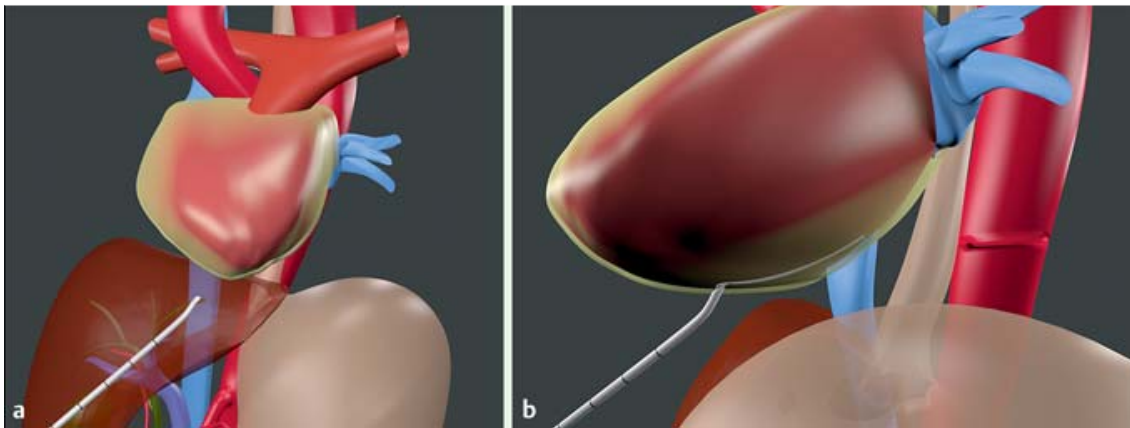

Fig. 1 a A subxiphoid puncture with a Tuohy needle is used to access the pericardial space for epicardial mapping and ablation. $\mathbf{b}$ Accessing the pericardial space with a guidewire.
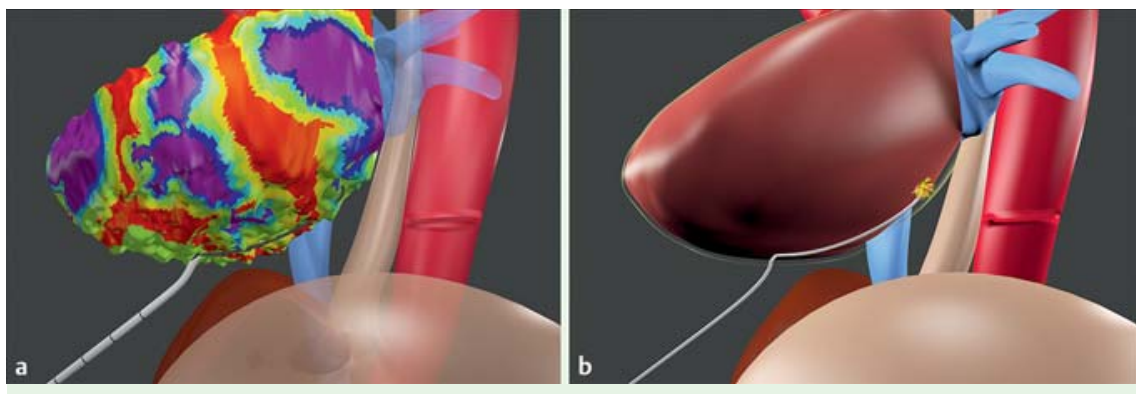

Fig.2 a Epicardial electrophysiological mapping. b Epicardial ablation.

Endocardial ablation has long been used to treat cardiac arrhythmias. However, the indications for epicardial intervention via the pericardial space have continuously increased since the technique was first described [1-3]. A subxiphoid puncture is used to access the pericardial space ( Fig. 1 a, $\bullet$ Fig. 1 b), allowing epicardial mapping and ablation ( $\bullet$ Fig. 2 a, - Fig.2b). Various complications have been described [4-8], which range from those caused by the puncture access (damage to the liver, colon, diaphragm, right ventricle, lungs) to those secondary to the ablation itself (damage to the coronary vessels, phrenic nerve, cardiac wall).

We present the case of a 63-year-old man admitted who had syncope, caused by transitory ventricular tachycardia secondary to Chagas disease with advanced chronic heart failure. The patient underwent epicardial ablation, which was complicated by the formation of an esophageal-mediastinal fistula, diagnosed with computed tomography and upper gastrointestinal endoscopy 15 days after the procedure.
Following a multidisciplinary consultation during which the patient's overall clinical status was considered, a conservative treatment combining thoracic drainage and the endoscopic placement of an overthe-scope clip was attempted ( $\bullet$ Video 1 ). The procedure was conducted without technical difficulty, and the patient left the intensive care unit on the next day. After 10 days, esophagography showed the clip still in place, with no detectable leakage of contrast medium. Liquid oral intake was initiated, and with progressive improvement in the patient's clinical status, he was discharged home 3 days later.

The patient returned on the 35th day after the endoscopic procedure for a follow-up examination. Endoscopy demonstrated that the metallic clip was no longer in place, but only a small pseudodiverticulum was visible, with no leakage of contrast medium detected on fluoroscopy.

\section{Endoscopy_UCTN_Code_TTT_1AO_2AI}

Competing interests: None

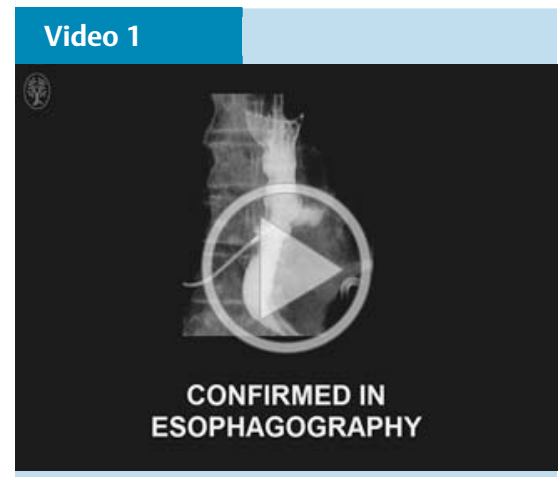

Conservative treatment combining thoracic drainage and the endoscopic placement of an over-the-scope clip to manage esophageal mediastinal fistula complicating epicardial ablation in a patient with Chagas disease.

\section{Eduardo Guimarães Hourneaux de Moura ${ }^{1}$, Gustavo Luís Rodela Silva ${ }^{1}$, Eduardo Turiani Hourneaux de Moura', Leonardo Zorrón Cheng Tao Pu ${ }^{1}$, Vinicius Leite de Castro ${ }^{1}$, Diogo Turiani Hourneaux de Moura', Rubens Antônio Aissar Sallum²}

1 São Paulo University Medical School, Gastrointestinal Endoscopy Unit, Gastroenterology Department, São Paulo, Brazil

2 Sao Paulo University Medical School, Gastroenterology/Esophageal Surgery, Sao Paulo, Brazil

\section{References}

1 Sosa E, Scanavacca M, d'Avila A et al. A new technique to perform epicardial mapping in the electrophysiology laboratory. J Cardiovasc Electrophysiol 1996; 7: 531-536

2 Sosa E, Scanavacca M. Images in cardiovascular medicine. Percutaneous pericardial access for mapping and ablation of epicardial ventricular tachycardias. Circulation 2007; 115: e542-e 544

3 Boyle NG, Shivkumar K. Epicardial interventions in electrophysiology. Circulation 2012; 126: 1752 - 1769

4 Sacher F, Roberts-Thomson K, Maury P et al. Epicardial ventricular tachycardia ablation a multicenter safety study. J Am Coll Cardiol 2010; 55: $2366-2372$

5 Koruth JS, Aryana A, Dukkipati SR et al. Unusual complications of percutaneous epicardial access and epicardial mapping and ablation of cardiac arrhythmias. Circ Arrhythm Electrophysiol 2011; 4: 882-888

6 Mathuria N, Buch E, Shivkumar K. Pleuropericardial fistula formation after prior epicardial catheter ablation for ventricular tachy- 
cardia. Circ Arrhythm Electrophysiol 2012; 5: e18-e19

7 Tschabrunn CM, Haqqani HM, Zado ES et al. Repeat percutaneous epicardial mapping and ablation of ventricular tachycardia: safety and outcome. J Cardiovasc Electrophysiol 2012; 23: $744-749$

8 Javaheri A, Glassberg HL, Acker MA et al. Constrictive pericarditis presenting as a late complication of epicardial ventricular tachycardia ablation. Circ Heart Fail 2012; 5: e22-e23

\section{Bibliography}

DOI http://dx.doi.org/

10.1055/s-0034-1393594

Endoscopy 2015; 47: E592-E593

(c) Georg Thieme Verlag KG

Stuttgart · New York

ISSN 0013-726X

\section{Corresponding author}

\section{Gustavo Luís Rodela Silva, MD}

São Paulo University Medical School

Gastrointestinal Endoscopy Unit

Gastroenterology Department

Av. Dr Eneas Carvalho de Aguiar, 255, 6th Floor São Paulo, SP

Brazil

Fax: +55-11-3887-7593

gustavoluisrodela@gmail.com 